\title{
Updating and Extending an UV/Vis Spectroscopy-Based Assay for Monitoring of Transformations Between Nucleosides and Nucleobases
}

\author{
Felix Kaspar, ${ }^{1,2, *}$ Robert T. Giessmann, ${ }^{1}$ Sarah Westarp, ${ }^{1,2}$ Katja F. Hellendahl, ${ }^{1}$ Niels Krausch, ${ }^{1}$ Isabel Thiele, ${ }^{1}$ Miriam C. \\ Walczak, ${ }^{1,2}$ Peter Neubauer, ${ }^{1}$ Anke Wagner ${ }^{1,2, *}$ \\ ${ }^{1}$ Technische Universität Berlin, Institute of Biotechnology, Chair of Bioprocess Engineering, Ackerstraße 76, D-13355 Berlin, \\ Germany \\ ${ }^{2}$ BioNukleo GmbH, Ackerstraße 76, D-13355 Berlin, Germany
}

In this report we update our recently described method for spectral unmixing-based reaction monitoring of nucleobase cleavage reactions. We present an extended suite of 38 established nucleoside substrates, recommendations regarding solutions to common problems, further applications and adaptations of the method that necessitate deviation from the previously reported protocol.

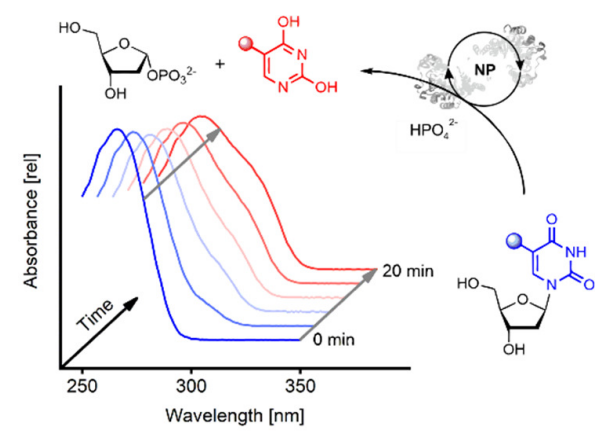

Nucleoside altering enzymes harbor significant potential for the synthesis of nucleoside analogs. Nucleoside phosphorylases (NPs), for instance, catalyze the reversible phosphorolytic cleavage of nucleosides into the corresponding free nucleobase and pentose-1-phosphate (Scheme 1) and are widely applied for the preparation of modified nucleosides. ${ }^{[1-11]}$ Consequently, their kinetic and thermodynamic characterization has attracted increased interest and demanded the development of efficient analytical tools. ${ }^{[12,13]}$

Recently, we reported a UV/Vis spectroscopy-based method for the monitoring of these reactions that largely eliminated the need for HPLC. ${ }^{[14]}$ For this method, we employed spectral unmixing to derive nucleoside/ nucleobase ratios from experimental UV absorption spectra based on suitable reference spectra. Implemented into the workflow of a high-throughput assay, this methodology facilitated a $>20$-fold reduction of data acquisition time and a roughly 5 -fold decrease in cost compared to conventional HPLC, while maintaining very comparable accuracy and excellent reproducibility. Unlike other non-HPLC-based methods, ${ }^{[15-27]}$ our approach offers a uniquely broad substrate spectrum, including all natural and several examples of modified nucleosides, as well as high adaptability and the straightforward application to any substrate without the need for laborious method development.

Following the initial report of our method, it has found wide-spread use in our laboratory and was successfully applied to several projects. Most notably, previous spectral characterization of a range of nucleoside substrates and their corresponding nucleobases enabled the investigation of the thermodynamic reaction control of nucleoside phosphorolysis. $^{[12]}$ Here we were able to measure slight temperature-induced changes of reaction equilibria of the phosphorolysis of 24 nucleosides that allowed convenient experimental access to thermodynamic properties of those reactions. Knowledge of the UV absorption spectra of nucleosides and nucleobases also enabled qualitative reaction monitoring of nucleoside transglycosylations to determine the time to equilibrium and reduce sampling effort. ${ }^{[11]}$ Further work to employ our method for the kinetic characterization of several NPs across their broad working space to probe the limits of their tolerance to harsh reaction conditions is currently underway. ${ }^{[28]}$ In addition, this method has greatly aided our efforts to explore the substrate spectra of other nucleobase-cleaving enzymes, ${ }^{[29]}$ empowered screening projects ${ }^{[30]}$ and overall alleviated our dependence on HPLC. ${ }^{[31]}$ Ultimately, these examples showcase the remarkable potential of our spectral unmixingbased method for high sample throughput and efficient monitoring of nucleobase cleavage reactions.

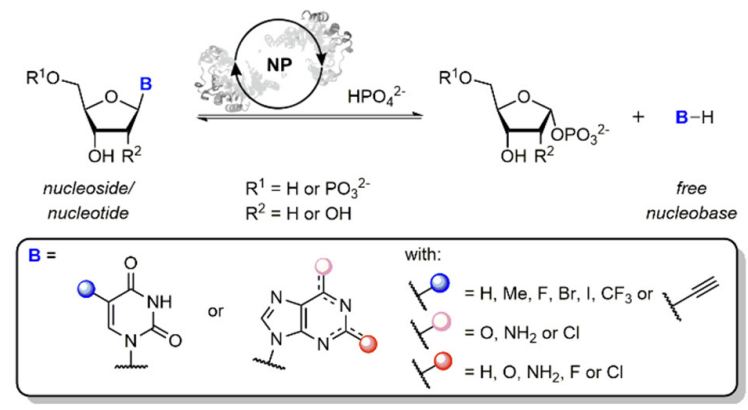

Scheme 1. Nucleoside/nucleotide phosphorolysis of pyrimidine or purine species. With two exceptions, all nucleobases featured in this report are described.

In our pursuit to broadly apply this approach we encountered several obstacles and had to find appropriate solutions. In this update we expand the scope of established substrates, share our experience and recommendations regarding solutions to common problems and describe some examples of alternative uses of the original method that necessitate deviation from the previously reported protocol. This contribution highlights the utility of spectral unmixing for the monitoring and analysis of (enzymatic) nucleobase cleavage reactions and will prove helpful to all current 

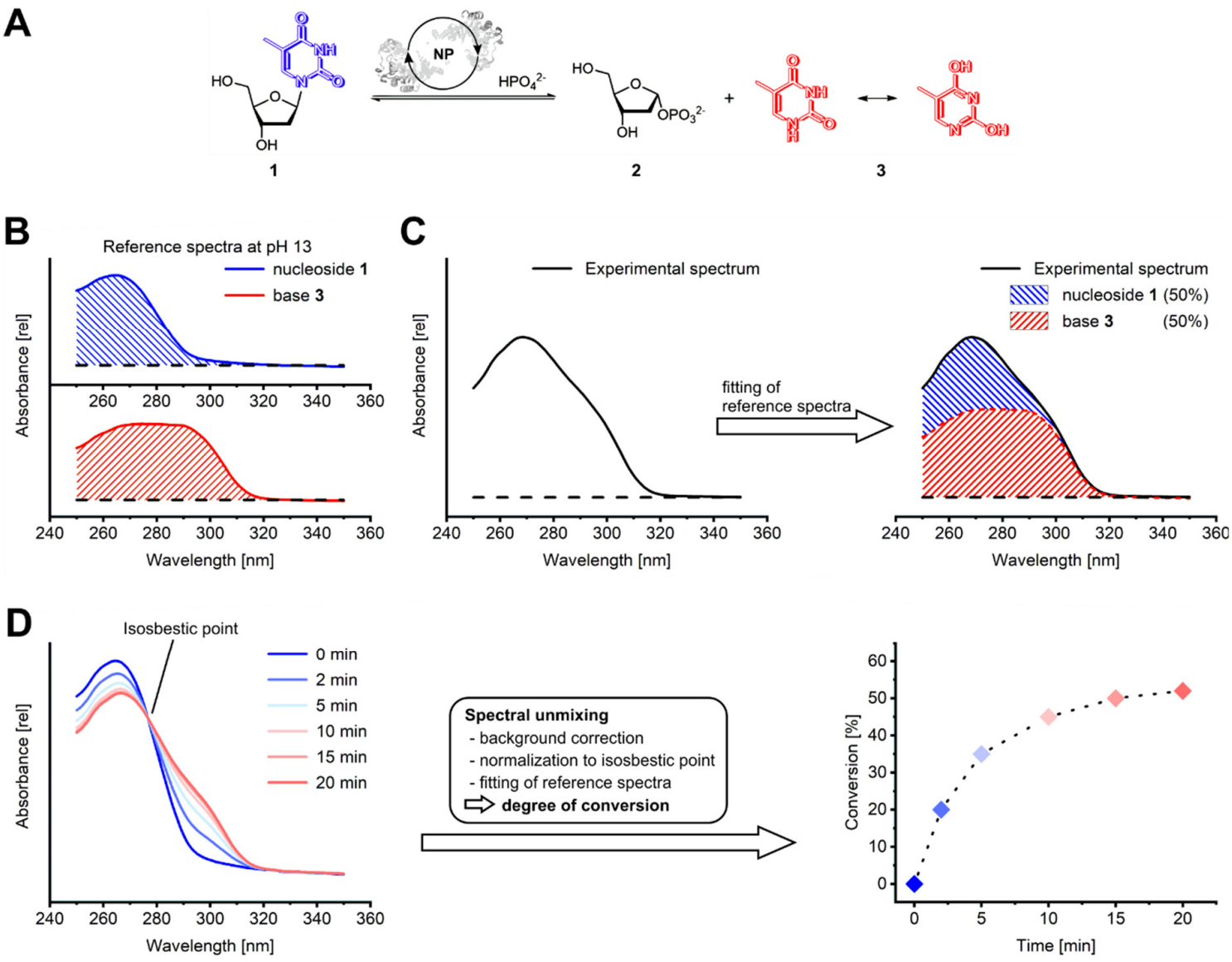

Figure 1. The principle of spectral unmixing-based reaction monitoring. A Enzymatic phosphorolysis of thymidine (1) into 2'deoxyribose-1-phosphate (2) and the free nucleobase 3. B The substrate $\mathbf{1}$ and product $\mathbf{3}$ of the reaction feature markedly different UV absorption spectra under alkaline conditions. $\mathbf{C}$ The spectra of $\mathbf{1}$ (blue) and $\mathbf{3}$ (red) can be fitted to an experimental spectrum (black line) obtained during a reaction to derive the individual contribution of both species to the observed spectrum (hashed areas). D Unmixing of multiple experimental spectra obtained during a reaction (left) enables reaction monitoring by deriving the degree of conversion at every sampled timepoint (right). Spectral unmixing of nucleoside transformations generally includes background correction, normalization to the isosbestic point of base cleavage and fitting of the respective reference spectra.

and future users of our previously published method. ${ }^{[14]}$

\section{The Principle of Reaction Monitoring via Spectral Unmixing}

Spectral unmixing in this case describes the concept of linear combination of absorption spectra that can be traced back to its individual components. In this sense, any mixture of two (or more) compounds with known absorption spectra can be deconvoluted into its constituents if appropriate reference spectra are available. ${ }^{[32]}$

Our method for monitoring of nucleobase cleavage reactions employs this concept by deriving nucleoside/nucleobase ratios from experimental spectra recorded after alkaline dilution of samples from a reaction mixture. ${ }^{[14]}$ Under alkaline conditions the UV absorption spectra of nucleosides and nucleobases (Figure 1A) differ sufficiently to allow discrimination (Figure 1B). Therefore, previously recorded reference spectra can be fitted to a background-corrected experimental spectrum (Figure 1C) to determine the contribution and ratio of its individual constituents (namely substrate and product of the reaction).
This approach allows for efficient reaction monitoring when multiple UV absorption spectra from a given reaction are available and can be deconvoluted into their individual components to derive the respective degree of conversion (Figure 1D). Conveniently, nearly all nucleosidenucleobase pairs display an isosbestic point of base cleavage that allows for normalization to correct for differences in signal intensity which in turn eliminates potential errors from pipetting inaccuracy. At the isosbestic point, the nucleoside and nucleobase in question possess the same extinction coefficient which manifests itself as a constant signal intensity at this wavelength throughout a reaction (see Figure 1D for the pair of $\mathbf{1}$ and $\mathbf{3}$ ).

In this workflow alkaline dilution of the sample serves a threefold purpose. This step simultaneously terminates the reaction by denaturing the enzyme, adjusts the concentration of the analytes and regulates the $\mathrm{pH}$ value of the sample to achieve deprotonation and spectral shifting of the UV absorption spectra. The suitable degree of alkaline dilution as well as the concentration of the base used for dilution (in our case aqueous $\mathrm{NaOH}$ ) varies between different nucleoside-nucleobase pairs, since the extinction 
coefficients and the $\mathrm{pH}$-range for stable and reproducible spectra needed for analysis differ. For example, purine nucleosides generally display a stronger UV absorption than pyrimidine nucleosides, which requires smaller sampling volumes to achieve the same peak signal intensity for these substrates.

\section{Updated List of Established Substrates}

Extending our previously reported list of 20 nucleosides, ${ }^{[14]}$ we herein present the spectral characteristics of 38 substrates (Table 1). Reference spectra for all compounds and their bases listed in Table 1 are freely available from the externally hosted supplementary material. ${ }^{[33]}$ The updated list of established substrates now includes several modified purine nucleosides (22-27 and 33-36) and highly modified pyrimidines such as 5-trifluoromethyluridine (15). Notably, we also characterized some 5'-phosphorlyated nucleotides and found their spectral properties to be essentially identical to their respective non-phosphorylated counterparts, conveniently allowing the reaction monitoring for these substrates without the need for additional reference spectra or method development.

Table 1. Spectral properties of nucleosides, nucleotides and their bases under alkaline conditions

\begin{tabular}{|c|c|c|c|c|c|c|}
\hline & Compound $^{[\mathrm{a}]}$ & $\mathrm{pH}^{[\mathrm{b}]}$ & $\begin{array}{c}\lambda_{\max } \\
\text { Nucleoside/ } \\
\text { Nucleotide }[\mathrm{nm}]\end{array}$ & $\begin{array}{c}\lambda_{\max } \\
\text { Nucleobase } \\
{[\mathrm{nm}]}\end{array}$ & $\begin{array}{c}\text { Isosbestic } \\
\text { Point of Base } \\
\text { Cleavage [nm] }\end{array}$ & $\begin{array}{c}\text { Spectral } \\
\text { Extension } \\
{[\mathrm{nm}]}\end{array}$ \\
\hline \multirow{17}{*}{ 窇 } & Uridine $(4)^{[\mathrm{c}]}$ & 13 & 262 & \multirow{2}{*}{281} & 271 & 310 \\
\hline & $2^{\prime}$-Deoxyuridine $(5)^{[\mathrm{c}]}$ & 13 & 262 & & 272 & 310 \\
\hline & 5-Methyluridine $(\mathbf{6})^{[\mathrm{c}]}$ & 13 & 267 & \multirow{2}{*}{290} & 277 & 320 \\
\hline & Thymidine $(\mathbf{1})^{[\mathrm{c}]}$ & 13 & 266 & & 278 & 320 \\
\hline & 5 -Fluorouridine $(7)^{[\mathrm{c}]}$ & 13.3 & 269 & \multirow{2}{*}{281} & 282 & 325 \\
\hline & 2'-Deoxy-5-fluorouridine $(\mathbf{8})^{[\mathrm{c}]}$ & 13.3 & 268 & & 280 & 325 \\
\hline & 5 -Bromouridine $(9)^{[\mathrm{c}]}$ & 13 & 276 & \multirow{2}{*}{290} & 283 & 330 \\
\hline & 5-Bromo-2'-deoxyuridine $(\mathbf{1 0})^{[\mathrm{c}]}$ & 13 & 275 & & 282 & 330 \\
\hline & 5-Iodouridine $(\mathbf{1 1})^{[\mathrm{c}]}$ & 13.3 & 281 & \multirow{2}{*}{291} & 283 & 340 \\
\hline & $2^{\prime}$-Deoxy-5-iodouridine $(\mathbf{1 2})^{[\mathrm{c}]}$ & 13.3 & 279 & & 282 & 340 \\
\hline & 5-Ethynyluridine $(\mathbf{1 3})^{[\mathrm{c}]}$ & 13.3 & 285 & \multirow{2}{*}{298} & 262,288 & 340 \\
\hline & 2'-Deoxy-5-ethynyluridine $(14)^{[\mathrm{c}]}$ & 13.3 & 284 & & 262,288 & 340 \\
\hline & 5-Trifluoromethyluridine (15) & 10 & 259 & 279 & 267 & 310 \\
\hline & Cytidine $(\mathbf{1 6})^{[\mathrm{c}]}$ & 13.7 & 271 & & 271 & 310 \\
\hline & $2^{\prime}$-Deoxycytidine $(\mathbf{1 7})^{[\mathrm{c}]}$ & 13.7 & 271 & & 271 & 310 \\
\hline & Uridine-5'-monophosphate (18) & 13 & 262 & 281 & 271 & 310 \\
\hline & Cytidine-5'-monophosphate (19) & 13.7 & 271 & 281 & 271 & 310 \\
\hline \multirow{21}{*}{ 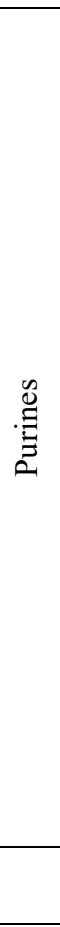 } & Adenosine $(\mathbf{2 0})^{[\mathrm{c}]}$ & 13 & 259 & \multirow{2}{*}{268} & 267 & 310 \\
\hline & $2^{\prime}$-Deoxyadenosine $(\mathbf{2 1})^{[\mathrm{c}]}$ & 13 & 259 & & 267 & 310 \\
\hline & 2-Fluoroadenosine (22) & 13 & 260 & \multirow{2}{*}{268} & 271 & 310 \\
\hline & 2'-Deoxy-2-fluoroadenosine (23) & 13 & 260 & & 271 & 310 \\
\hline & 2-Chloroadenosine (24) & 13 & 264 & \multirow{2}{*}{271} & 271 & 310 \\
\hline & 2-Chloro-2'-deoxyadenosine (25) & 13 & 264 & & 271 & 310 \\
\hline & 2-Aminoadenosine (26) & 13 & 279 & \multirow{2}{*}{284} & 285 & 320 \\
\hline & 2-Amino-2'-deoxyadenosine (27) & 13 & 279 & & 285 & 320 \\
\hline & Guanosine $(\mathbf{2 8})^{[\mathrm{c}]}$ & 13 & 264 & \multirow{2}{*}{273} & 279 & 310 \\
\hline & $2^{\prime}$-Deoxyguanosine $(\mathbf{2 9})^{[\mathrm{c}]}$ & 13 & 264 & & 279 & 310 \\
\hline & Inosine $(\mathbf{3 0})^{[\mathrm{c}]}$ & 13 & 252 & \multirow{2}{*}{262} & 263 & 320 \\
\hline & $2^{\prime}$-Deoxyinosine $(\mathbf{3 1})^{[\mathrm{c}]}$ & 13 & 252 & & 263 & 320 \\
\hline & Xanthosine (32) & 13.3 & 276 & 282 & 276 & 320 \\
\hline & 2,6-Dichloropurine riboside (33) & 9 & 274 & \multirow{2}{*}{279} & 278 & 310 \\
\hline & 2,6-Dichloro 2'-deoxyriboside (34) & 9 & 274 & & 278 & 310 \\
\hline & $\begin{array}{l}\text { 6-Chloro-2-fluoropurine riboside } \\
\text { (35) }\end{array}$ & 9 & 269 & \multirow{2}{*}{273} & 271 & 310 \\
\hline & $\begin{array}{l}\text { 6-Chloro-2-fluoropurine 2'- } \\
\text { deoxyriboside (36) }\end{array}$ & 9 & 269 & & 271 & 310 \\
\hline & Adenosine-5'-monophosphate (37) & 13 & 259 & 268 & 267 & 310 \\
\hline & Guanosine-5'-monophosphate (38) & 13 & 264 & 273 & 279 & 310 \\
\hline & Inosine-5'-monophosphate (39) & 13 & 252 & 262 & 263 & 320 \\
\hline & $\begin{array}{l}\text { 1,2,4-Triazole-3-carboxamide } \\
\text { riboside }(\mathbf{4 0})^{[\mathrm{d}]}\end{array}$ & 13 & $-[e]$ & {$[\mathrm{e}]$} & _[e] & _[e] \\
\hline
\end{tabular}

[a] Please see the Supporting Information (Figure S1) for the structures of all compounds, [b] pH 9 was generally achieved in $50 \mathrm{mM}$ Tris/NaOH buffer, pH 10 in $100 \mathrm{mM}$ glycine/NaOH buffer, pH 13 in $100 \mathrm{mM} \mathrm{NaOH}, \mathrm{pH} 13.3$ in $200 \mathrm{mM} \mathrm{NaOH}$ and $\mathrm{pH} 13.7$ in $500 \mathrm{mM} \mathrm{NaOH}$, [c] From the original report, ${ }^{[14]}$ [d] Ribavirin, [e] Both $\lambda_{\max }$ values are at $<250 \mathrm{~nm}$ and there is no isosbestic point of base cleavage. Note that reaction monitoring can still be performed via single- or multiwavelength detection but normalization to the isosbestic point of base cleavage is not possible for this substrate. 
Dealing with Background

The most common obstacle with the presented method is background absorption. Different types of background absorption are typically observed and need to be addressed individually (Figure 2). Please note that while we use the phosphorolysis of thymidine (1, Figure 2A) as an exemplary reaction in this manuscript, the same principles translate to all nucleosides and nucleotides and can be applied in the same manner.

An occasional and unavoidably type of background signal is atypical UV-absorption of the multiwell plate or of particles. A typical background signal is in the range of 0.03 absorption units (AU) at $300 \mathrm{~nm}$ and curves up towards slightly higher intensities at $250 \mathrm{~nm}$ (Figure 2B). This background is very well reproducible and easily adjusted for, as described in our original method. However, on average in approximately $1-2 \%$ of all measurements, we observed increased absorption across the entire spectrum apparent as a distinct baseline shift (Figure 2C). This results in an inability to obtain accurate fits without manual spectral processing (which we choose to explicitly abstain from), as in these cases the baseline can be shifted $>0.10 \mathrm{AU}$. The straightforward solution in this case is remeasurement of the sample in a different well, which generally resolves the problem.

We did not mention correction for protein background in our initial report. That is because in most cases enzyme background absorption in the considered range of 250 $350 \mathrm{~nm}$ is quasi indetectable when using protein concentrations of $50 \mu \mathrm{g} / \mathrm{mL}$ or less in the reaction mixture that is sampled. In selected instances where either higher concentrations and/or especially UV-active enzymes are used, appropriate background correction of the experimental sample may be necessary (Figure 2D). This can easily be carried out with a spectrum of a suitably diluted sample of the enzyme. Note that one may keep using the previously obtained reference spectra for the substrate and product without any further alterations as those are already corrected for their respective background.

Background absorption by UV-active components in the reaction mixture needs to be addressed on a case-to-case basis. Generally, all common buffers, protein stabilizing agents or even artifacts from protein purification such as imidazole do not represent any challenge to the method and allow straightforward use of the standard procedure without any additional background correction. When using some organic cosolvents, however, we noticed significant background absorption in the lower wavelength region. Whereas alcohols including methanol, ethanol, isopropanol, ethylene glycol and glycerol may be used without alterations to the method (see Supporting information for details), solvents like dimethyl sulfoxide (DMSO) and dimethyl formamide (DMF) create background signals that need to be accounted for (Figure 2E). In these cases we found success employing background spectra that reflect the specific content of UV-active solvent in the sample and, if appropriate, limiting the wavelength range for fitting of pyrimidine UV absorption spectra to the information-rich tail region (i.e. $265-295 \mathrm{~nm}$ for uridine, 4). We even found success when using especially UV-active reaction components, such as dithiothreitol (DTT), that proved problematic in some instances, by selecting appropriate substrates and selectively limiting the fitting range (please see Supporting Information for details and Figure S3 for an example).

In rare cases we observed elevated baselines when using very concentrated buffers for dilution and spectral measurement. Again, appropriate background spectra to correct for this shift succeeded in resolving this issue.

Nonetheless, it should be stressed that background correction does not always lead to accurate and reliable data. We experienced serious difficulties to correct for noise in instances where the background signal intensity is comparable to the signal intensity of the nucleosidenucleobase pair under investigation and directly and/or completely overlaps with this signal (i.e. when examining the effect of enzyme inhibitors in stoichiometric quantities). In principle, background subtraction from the experimental spectrum is still possible and yields a processed spectrum that can be fitted, but fit quality and, consequently, accuracy of this approach suffered tremendously. We ascribed this to the fact that the signal intensity of the dynamic analytes (substrate and product) is largely irrelevant as those values are normalized to the isosbestic point and considered only in relation to one another, but background signals from most sources are absolute quantities and thus vary with and are very sensitive to pipetting accuracy. As a rule of thumb, we recommend our method for cases where background absorption does not exceed $20 \%$ of the relevant signals (i.e. signal-to-noise ratio should remain $>5$ ).

\section{Reactant Instability}

A critical factor for any method is stability and detectability of the analytes. While all nucleosides and nucleobases in our original report displayed excellent stability towards the quenching and analysis conditions, we noted some issues within the extended substrate range. Fluorinated purine nucleosides $\mathbf{2 2}$ and $\mathbf{2 3}$ were found to be quite sensitive to alkaline conditions as these nucleosides underwent a temperature- and base-promoted side reaction (Figure S2), presumably by 5 ' $-\mathrm{OH}$ attack at the purine ring. We were able to bypass this issue by quick sample processing avoiding any unnecessary storage. At room temperature and pH 13, compounds $\mathbf{2 2}$ and $\mathbf{2 3}$ remained stable enough for analysis for at least $10 \mathrm{~min}$.

\section{Further applications of the method}

While the original protocol has proven to be a robust and versatile method, we have used spectral unmixing-based reaction monitoring in instances that deviated from the original conditions. Some applications that necessitated adjusted sample treatment are worth mentioning.

In our earlier report we have used purified protein in all reactions. ${ }^{[14]}$ The subsequently published applications also featured pure protein in all instances. ${ }^{[12]}$ However, the use of unpurified protein, e.g. in the form of crude cell lysate, is highly desirable for screening of mutants or whole-cell reactions. We were pleased to find that even crude protein preparations (as lysed cells or cell-free extract) permitted the use of spectral unmixing-based reaction monitoring, if appropriate background correction is considered and the background signal remains within a manageable range (see above and Supporting Information). 
A

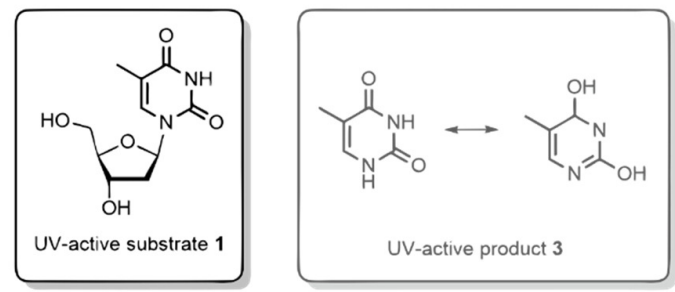

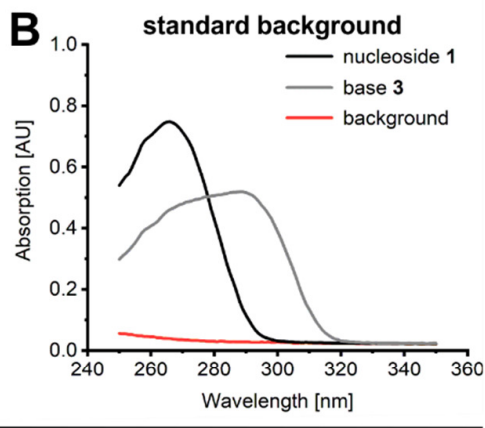

$\mathbf{E}$

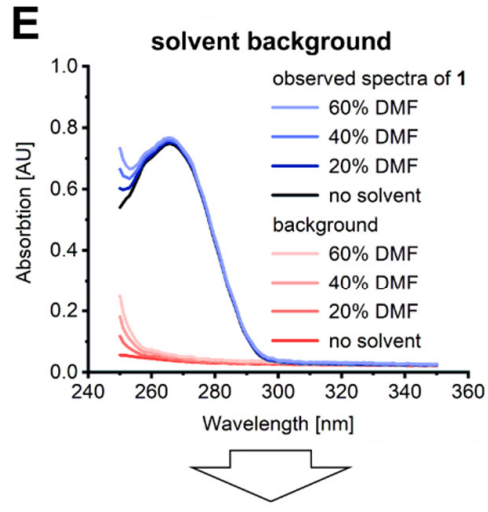

correct for background consider limiting wavelength range for fitting
D

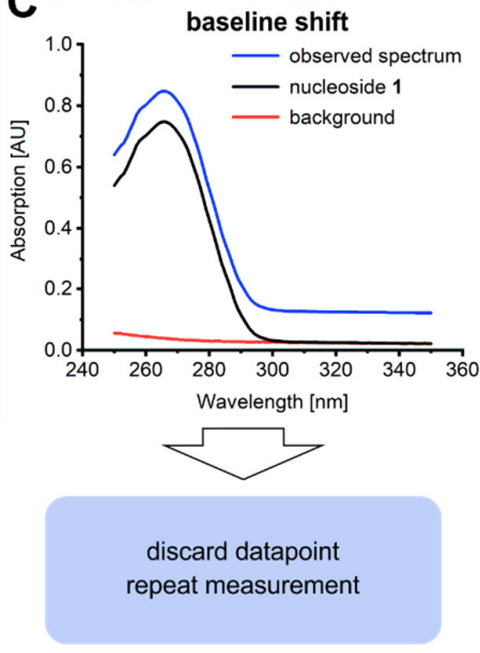

correct for background consider using less protein

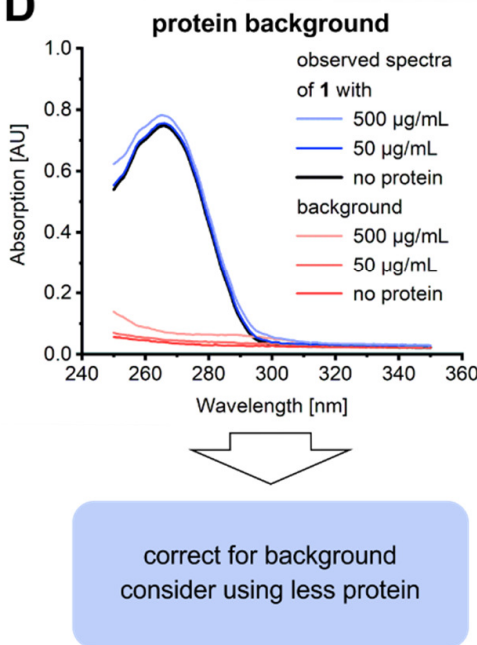

Figure 2. Common background signals. A Exemplary UV-active reactants 1 and 3. B Standard background observed from the absorption of the 96-well plate filled with water or aqueous $\mathrm{NaOH}$. Signals for $\mathbf{1}$ and $\mathbf{3}$ represent typical signal intensities observed with reactions with $2 \mathrm{mM}$ nucleoside substrate and a dilution factor of 15 during sampling. $\mathbf{C}$ Baseline shift observed in approximately $1-2 \%$ of measurements. D Background observed in reactions with significant protein content. Purified Escherichia coli thymidine phosphorylase was used to recreate typical protein backgrounds by 15 -fold dilution in $100 \mathrm{mM}$ $\mathrm{NaOH}$. Note that some proteins may cause significantly more or less background. E Representative background observed with some organic solvents. Dimethyl formamide (DMF) was diluted 10-fold in $100 \mathrm{mM} \mathrm{NaOH}$ to record the background signals.

In cases where heterogenous reactions were applied, centrifugation of the quenched alkaline samples prior to analysis was necessary and successfully reduced background noise and baseline shifts caused by particles.

Conveniently, spectral shifting of the UV absorption spectra of the free nucleobases doesn't always require application of a strong base. Selected nucleosidenucleobase pairs feature a marked spectral shift and stable spectra in a pH region easily accessible by established buffer systems (e.g. 33-36, Table 1). This also presents an opportunity to monitor live reactions, either by applying a continuous assay or discontinuously monitoring very slow reactions by diluting samples of the reaction mixture in appropriate buffer (see Supporting Information for details).

Some nucleosides precluded application of the original protocol that involves quenching of reaction samples in aqueous $\mathrm{NaOH}$. Chlorinated scaffolds 33-36 display remarkably dynamic UV absorption spectra at $\mathrm{pH}$ values above 11 (Figure S4) and we were unable to obtain reproducible spectral fits using alkaline quenching. Fortunately, this issue could be resolved by employing organic solvents like methanol as an alternative quenching medium and subsequently adjusting the $\mathrm{pH}$ value to 9 for analysis (see Supporting Information for details). A similar methodology featuring a different buffer system succeeded for the trifluorinated pyrimidine $\mathbf{1 5}$ (Table 1, Figure S6).

These examples only present a snapshot of the diverse applications of spectral unmixing-based reaction monitoring of transformations between nucleosides and nucleobases that one may envision. Nonetheless, we are confident that the lessons learned thus far will translate well to other scenarios, reaction systems, enzymes and applications where similar issues might be encountered.

\section{Concluding Remarks}

Spectral unmixing presents a powerful tool for the efficient reaction monitoring of nucleobase cleavage reactions, with nucleoside phosphorolysis representing a highly relevant example. Spectral unmixing of UV absorption spectra conveniently allows for increased sample throughput compared to other methods and doesn't require expensive equipment or reagents. We have employed this method extensively and demonstrated its precision, versatility, robustness and ease of handling. The present report extends the range of established substrates and discusses common problems and notable modifications to the original protocol. Reference spectra for all substrates and nucleobases listed in this article ${ }^{[33]}$ as well as our Python code used for spectral 
unmixing ${ }^{[34,35]}$ can be obtained from an external online repository and we are happy to assist with their use. While specific scenarios may require evaluation and troubleshooting on a case-to-case basis, the strategies discussed herein will facilitate the straightforward and versatile application of this method.

\section{Associated Content}

The Supporting Information (PDF file) contains the author contributions and additional figures $\mathrm{S} 1-\mathrm{S} 7$ (including the structures of all nucleosides/nucleotides in this report: $\mathbf{1}$ and 4-40, as well as supplementary details, notes and flowcharts for experimental protocols).

\section{Author Information \\ Corresponding Authors \\ *Felix Kaspar, felix.kaspar@web.de, orcid.org/0000-0001-6391-043X \\ *Dr. Anke Wagner, anke.wagner@tu-berlin.de, orcid.org/0000-0001-6919-725X}

\section{Other Authors}

Robert T. Giessmann, orcid.org/0000-0002-0254-1500

Sarah Westarp, orcid.org/0000-0001-6498-8810

Katja F. Hellendahl, orcid.org/0000-0002-5408-3679

Niels Krausch, orcid.org/0000-0003-2325-6001

Dr. Peter Neubauer, orcid.org/0000-0002-1214-9713

\section{Keywords}

UV/Vis spectroscopy, spectral unmixing, reaction monitoring, nucleosides, nucleotides, nucleobases, nucleoside phosphorylases

\section{Acknowledgements}

This work was funded by the Deutsche Forschungsgemeinschaft (DFG, German Research Foundation) under Germany's Excellence Strategy-EXC 2008/1-390540038. We are grateful for the support of R.T.G. by the Einstein Foundation Berlin (ESB)-Einstein Center of Catalysis $\left(\mathrm{EC}^{2}\right)$. K.F.H. is funded by DFG grant no. 392246628 .

\section{Conflict of Interest}

A.W. is the CEO, P.N. is a board member, and F.K. is a scientific researcher in the biotech company BioNukleo $\mathrm{GmbH}$. The authors declare no competing financial interest.

\section{Experimental}

\section{General Remarks}

All chemicals were purchased from Sigma Aldrich, TCI, Carl Roth, Carbosynth or BioNukleo $\mathrm{GmbH}$ at the highest available quality and used without prior purification. Solutions of all compounds were prepared in water deionized to $18.2 \mathrm{M} \Omega \cdot \mathrm{cm}$ with a Werner water purification system. $\mathrm{NaOH}$ solutions were prepared with deionized water. Enzymatic reactions were typically prepared from stock solutions of substrates and buffer and started via the addition of enzyme. Various NPs were used, including among others Y01, Y02, N01 and N02 from BioNukleo $\mathrm{GmbH}$, Escherichia coli uridine and thymidine phosphorylase and purine NP and Bacillus subtilis pyrimidine NP, as described previously. ${ }^{[12]} \mathrm{UV}$ absorption spectra were recorded on a BioTek PowerWave HT plate reader, using UV/Vis-transparent 96-well plates (UV-STAR F-Bottom \#655801, Greiner Bio-One). Spectral processing, unmixing and data generation were performed as described previously ${ }^{[14]}$ with software freely available online. ${ }^{[34,35]}$ All data presented in this report and the Supporting Information are freely available from an external online repository. ${ }^{[33]}$

\section{Reference Spectra and Reaction Sampling}

Reference spectra for pure compounds were prepared from $2 \mathrm{mM}$ solutions by $10-20$-fold dilution in aqueous $\mathrm{NaOH}$. Reactions with purine or pyrimidine nucleosides were typically performed with either $1 \mathrm{mM}$ or $2 \mathrm{mM}$ of UV-active compounds. From the $2 \mathrm{mM}$ reactions, $20 \mu \mathrm{L}$ (purines) or $30 \mu \mathrm{L}$ (pyrimidines) were withdrawn and quenched in $\mathrm{NaOH}$ to give a final volume of $500 \mu \mathrm{L}$. From the $1 \mathrm{mM}$ reactions, twice as much sample volume was withdrawn and treated analogously. Note that exact adherence to these volumes is not necessary, as normalization to the isosbestic point accounts for differences in signal intensity. For halogenated nucleosides 33-36, typically, samples of $20 \mu \mathrm{L}$ were withdrawn and either diluted in $50 \mathrm{mM}$ Tris/ $\mathrm{NaOH}$ buffer ( $\mathrm{pH} 9$ ) to a volume of $500 \mu \mathrm{L}$ or quenched in an equal volume of $\mathrm{MeOH}$ or $\mathrm{iPrOH}$ before dilution in $50 \mathrm{mM}$ Tris/ $\mathrm{NaOH}$ buffer to give a final volume of $500 \mu \mathrm{L}$. Similarly, the fluorinated pyrimidine $\mathbf{1 5}$ was sampled by quenching in $\mathrm{PrOH}$ or $\mathrm{MeOH}$ followed by dilution with $100 \mathrm{mM}$ glycine/ $\mathrm{NaOH}$ buffer $(\mathrm{pH} 10)$ to a final volume of $500 \mu \mathrm{L}$. Subsequently, $200 \mu \mathrm{L}$ of the diluted alkaline samples were transferred to wells of a UV/Vis-transparent 96-well plate to record the UV absorption spectra. All spectra were recorded from $250-350 \mathrm{~nm}$ in steps of $1 \mathrm{~nm}$. For exact sampling procedures and example reactions, please see our earlier reports ${ }^{[12,14]}$ and the Supporting Information.

\section{References}

[1] S. Kamel, H. Yehia, P. Neubauer, A. Wagner, in Enzym. Chem. Synth. Nucleic Acid Deriv. (Ed.: F. J.), 2018, 1-28.

[2] S. Kamel, M. Weiß, H. F. T. Klare, I. A. Mikhailopulo, P. Neubauer, A. Wagner, Mol. Catal. 2018, 458, 52-59.

[3] I. V Fateev, K. V Antonov, I. D. Konstantinova, T. I. Muravyova, F. Seela, R. S. Esipov, A. I. Miroshnikov, I. A. Mikhailopulo, Beilstein J. Org. Chem. 2014, 10, 1657-1669.

[4] H. Komatsu, T. Araki, Nucleosides. Nucleotides Nucleic Acids 2005, 24, 1127-1130.

[5] I. D. Konstantinova, K. V Antonov, I. V Fateev, A. I. Miroshnikov, V. A. Stepchenko, A. V Baranovsky, I. A. Mikhailopulo, Synthesis. 2011, 1555-1560.

[6] M. Rabuffetti, T. Bavaro, R. Semproli, G. Cattaneo, M. Massone, F. C. Morelli, G. Speranza, D. Ubiali, Catalysts 2019, 9, 355.

[7] H. Yehia, S. Westarp, V. Röhrs, F. Kaspar, T. R. Giessmann, F. T. H. Klare, K. Paulick, P. Neubauer, J. Kurreck, A. Wagner, Molecules 2020, 25, 934 .

[8] B. Z. Eletskaya, D. A. Gruzdev, V. P. Krasnov, G. L. Levit, M. A. Kostromina, A. S. Paramonov, A. L. Kayushin, I. S. Muzyka, T. I. Muravyova, R. S. Esipov, et al., Chem. Biol. Drug Des. 2019, 93, 
$605-616$.

[9] X. Zhou, K. Szeker, B. Janocha, T. Böhme, D. Albrecht, I. A. Mikhailopulo, P. Neubauer, FEBS J. 2013, 280, 1475-1490.

[10] K. Szeker, X. Zhou, T. Schwab, A. Casanueva, D. Cowan, I. A. Mikhailopulo, P. Neubauer, J. Mol. Catal. B Enzym. 2012, 84, 27-34.

[11] F. Kaspar, R. T. Giessmann, K. Hellendahl, P. Neubauer, A. Wagner, M. Gimpel, ChemBioChem 2020.

[12] F. Kaspar, R. T. Giessmann, P. Neubauer, A. Wagner, M. Gimpel, Adv. Synth. Catal. 2020, 362, 867-876.

[13] R. T. Giessmann, N. Krausch, F. Kaspar, N. M. Cruz Bournazou, A. Wagner, P. Neubauer, M. Gimpel, Processes 2019, 7, 380.

[14] F. Kaspar, R. T. Giessmann, N. Krausch, P. Neubauer, A. Wagner, M. Gimpel, Methods Protoc. 2019, $2,60$.

[15] E. W. Yamada, Methods Enzymol. 1978, 51, 423431.

[16] D. Ubiali, S. Rocchietti, F. Scaramozzino, M. Terreni, A. M. Albertini, R. Fernández-Lafuente, J. M. Guisán, M. Pregnolato, Adv. Synth. Catal. 2004, 346, 1361-1366.

[17] D. Singh, R. M. Schaaper, A. Hochkoeppler, Anal. Biochem. 2016, 496, 43-49.

[18] K. J. Seamon, J. T. Stivers, J. Biomol. Screen. 2015, 20, 801-809.

[19] M. Surette, T. Gill, S. MacLean, Appl. Environ. Microbiol. 1990, 56, 1435-1439.

[20] A. Stachelska-Wierzchowska, J. Wierzchowski, M. Górka, A. Bzowska, B. Wielgus-Kutrowska, Molecules 2019, 24, 1493.

[21] A. Bzowska, E. Kulikowska, D. Shugar, Pharmacol. Ther. 2000, 88, 349-425.

[22] N. G. Panova, E. V Shcheveleva, C. S. Alexeev, V. G. Mukhortov, A. N. Zuev, S. N. Mikhailov, R. S. Esipov, D. V Chuvikovsky, A. I. Miroshnikov, Mol. Biol. 2004, 38, 770-776.

[23] J. Wierzchowski, A. Stachelska-Wierzchowska, B. Wielgus-Kutrowska, A. Bzowska, Curr. Pharm. Des. 2017, 23, 6948-6966.

[24] J. Wierzchowski, M. Ogiela, B. Iwańska, D. Shugar, Anal. Chim. Acta 2002, 472, 63-74.

[25] J. Wierzchowski, A. Stachelska-Wierzchowska, B. Wielgus-Kutrowska, G. Mikleušević, Anal. Biochem. 2014, 446, 25-27.

[26] A. Stachelska-Wierzchowska, J. Wierzchowski, A. Bzowska, B. Wielgus-Kutrowska, Nucleosides, Nucleotides and Nucleic Acids 2018, 37, 89-101.

[27] D. J. Porter, J. Biol. Chem. 1992, 267, 7342-7351.

[28] F. Kaspar, P. Neubauer, A. Wagner, 2020, in preparation.

[29] S. Kamel, C. Walczak, P. Neubauer, A. Wagner, $\mathbf{2 0 2 0}$, in preparation.

[30] S. Westarp, J. Schollmeyer, P. Neubauer, A. Wagner, 2020, in preparation.

[31] K. F. Hellendahl, F. Kaspar, P. Neubauer, A. Wagner, 2020, in preparation.

[32] C. Quintano, A. Fernández-Manso, Y. E. Shimabukuro, G. Pereira, Int. J. Remote Sens. 2012, 33, 5307-5340.
[33] F. Kaspar, 2020, DOI 10.5281/zenodo.3716126.

[34] R. T. Giessmann, N. Krausch, 2019, DOI 10.5281/zenodo.3243376.

[35] R. T. Giessmann, https://gitlab.com/rgiessmann/data_toolbox 